\title{
Tungsten oxide electrode for measurement of ultralow liquid flow velocity
}

\author{
Yizhang Wen, Xiaoping Wang*, Peijun Cai, Bing Zhang \\ State Key Laboratory of Modern Optical Instrumentation, Department of Optical Engineering, \\ Zhejiang University, Hangzhou 310027, China \\ *Corresponding author \\ E-mail address: xpwang@zju.edu.cn \\ Fax: +86571 87951185
}




\section{Abstract:}

This study demonstrated the application of a tungsten oxide electrode to the measurement of ultralow liquid flow velocity based on streaming potential, using a $\mathrm{pH}$ glass electrode and temperature sensor to compensate for changes in open circuit potential induced by $\mathrm{pH}$ and temperature, respectively. A change in flow velocity on the surface of the tungsten oxide electrode caused the expected shift in its open circuit potential. Experimental results showed that the open circuit potential was linear relative to the logarithmic flow velocity, and the flow sensor exhibited excellent reproducibility and a large dynamic range, with the ratio between measurable maximum and minimum flow velocity surpassing 60:1. Moreover, a higher sensitivity was observed in the alkaline solution relative to the acidic solution, indicating that the alkaline solution is more suitable for this type of measurement. In conclusion, the tungsten oxide flow sensor provides a cheap, simple, adaptable, and energy-saving solution for the measurement of ultralow liquid flow velocity.

Keywords: ultralow flow velocity; tungsten oxide electrode; streaming potential; $\mathrm{pH}$. 


\section{Introduction}

Flow measurement is an important issue in industrial and agricultural production, national defense, trade, and various aspects of people's livelihood. Currently, electromagnetic [1], ultrasonic [2], differential pressure [3], vortex [4, 5], turbine [6], and thermal [7]flowmeters are the main sensing instruments adopted for the flow measurement.

The flow velocities of liquids used in the semiconductor industry and chemical analyses are usually very small. However, conventional flowmeters listed above usually cannot measure low flow velocity because of inadequate sensitivity, and they also do not have sufficient long-term stability. Therefore, there is a strong demand for a new sensor that can measure low flow velocities of liquids.

Many sensors have been developed in order to measure very low flow velocity because of demand in the semiconductor, medical, and chemical industries. Takamoto et al. improved the ultrasonic flowmeter so that it could reliably measure a flow rate of less than $1 \mathrm{~mL} / \mathrm{min}$ with a standard deviation of approximately $0.01 \mathrm{~mL} / \mathrm{min}$ in a glass tube with diameter of $0.5 \mathrm{~mm}$ [8].Some thermal flow velocimeters, which comprise heater elements and temperature sensors, have demonstrated a capability for measuring low liquid flow velocities. For instance, Dinh et al. used a hotwire to measure a low flow rate of $0.05 \mathrm{~mL} / \mathrm{min}$; in this flow channel, the flow velocity was lower than $3 \mathrm{~mm} / \mathrm{s}$ [9]. A flow velocity measurement method using the photothermal effect has been developed to realize a small, noninvasive microflow velocimeter, with a dynamic range of $25-300 \mu \mathrm{L} / \mathrm{min}$ in a microchannel; the minimum flow velocity 
that could be measured was $\sim 3 \mathrm{~cm} / \mathrm{s}$ [10]. There are other types of microflow measurement methods, including microparticle imaging velocimetry, in which flow velocities are obtained from the motion of tracers dispersed in the fluid; a bulk velocity of approximately $50 \mu \mathrm{m} / \mathrm{s}$ could be measured [11]. However, the tracer particles must be introduced into the solution for the flow measurement and they can be a disturbance to chemical reactions. Another technology uses an integrated micro-opto-fluidic flow-sensor, in which a fiber cantilever transduces flow rate into optical transmission, that could measure a minimum flow rate of $\sim 7 \mu \mathrm{L} / \mathrm{min}$ [12]. An ultrathin curved-up microcantilever flow sensor has been developed and calibrated for measurement of small flow velocities based on the changes in electrical parameters induced by pressure; experimental results showed that the microcantilever could measure flow velocities in the range of $0-23 \mathrm{~cm} / \mathrm{s}$ [13]. Laser systems using fiber Bragg gratings and doped fibers have been developed for use as sensors capable of measuring flow rates in a linear range from 0 to $200 \mathrm{~mL} / \mathrm{s}$, with a resolution of approximately $8 \mathrm{~mL} / \mathrm{s}$ in a pipe with diameter of $15 \mathrm{~mm}$ [14].

Although these methods meet the requirement of measuring low flow velocities, ultralow flow velocities could not be measured reliably, and most of these methods require complex systems and data processing. Meanwhile, efficient, stable measurement of ultralow flow velocity in open channels remains a challenge.

Park and Choa [15] have reported measurement of liquid flow rate by self-generated electrokinetic potential on the microchannel surface of a solid. In this paper, we propose the use of a tungsten oxide electrode as an indicator electrode and 
an $\mathrm{Ag} / \mathrm{AgCl}$ electrode as a reference electrode to sensor ultralow liquid flow velocities based on streaming potential. As the core of flow sensor, the tungsten oxide electrode has a bilayer structure consisting of a compact inner layer of $\mathrm{WO}_{3}$ and a loosely bound outer hydrated layer of $\mathrm{WO}_{3} \cdot \mathrm{xH}_{2} \mathrm{O}$. Lillard et al. has reported a corresponding decrease in the thickness of the surface layer under hydrodynamic effect [16]. In addition, our previous work demonstrated a dramatic change in the open circuit potential of the tungsten oxide electrode under the hydrodynamic effect. The new open circuit potential of the tungsten oxide electrode could remain stable under the fixed rotation speed of a magnetic rotor, gradually recovering when the magnetic stirrer was turned off. Repeated cycles revealed that tungsten oxide could maintain its stability under hydrodynamic conditions where oxide dissolution had altered the potential signals [17]. Thus, the tungsten oxide electrode could be used to measure liquid flow velocity. The responses of open circuit potentials to flow velocities were studied in solutions of different $\mathrm{pH}$. Therefore, to obtain accurate flow velocity, a $\mathrm{pH}$ glass electrode and a temperature sensor were used to compensate for changes in open circuit potential induced by $\mathrm{pH}$ and temperature.

\section{Materials and methods}

\subsection{Chemicals and reagents}

In this study, $0.05 \mathrm{M}$ potassium acid phthalate $(\mathrm{pH}=4), 0.025 \mathrm{M}$ mixed phosphate $(\mathrm{pH}=6.86), 0.01 \mathrm{M}$ borax $(\mathrm{pH}=9.18)$ and dilute hydrochloric acid were used as measuring solutions. All solutions were prepared by using deionized water (electric resistance of 18.2 MX) supplied by a MilliQ water purification system 
(Millipore, Billerica, MA).

\subsection{Electrode preparation}

A Teflon-coated tungsten electrode with a diameter of $2 \mathrm{~mm}$ (purity $>99.99 \%$ ) was purchased from Tianjin Aidahengsheng Technology Corporation (China). Then, the tungsten electrode was abraded with grinding paper, finely polished with $0.5-\mu \mathrm{m}$ aluminum oxide particles, and finally rinsed with distilled water. For producing the tungsten oxide electrode, we electrochemically oxidized the tungsten electrode in a $0.1 \mathrm{M} \mathrm{H}_{2} \mathrm{SO}_{4}$ solution by cycling the potential between 1 and $2 \mathrm{~V}$ vs. a saturated calomel electrode for 20 cycles at a scan rate of $20 \mathrm{mv} / \mathrm{s}$. Subsequently, the electrode was immersed in the same solution for $12 \mathrm{~h}$, washed with distilled water, and dipped in a buffer solution of $\mathrm{pH} 6.86[17,18]$.

\subsection{Experimental setup and signal acquisition}

The sensor section of the system consisted of a tungsten oxide electrode, a $\mathrm{pH}$ glass electrode, an $\mathrm{Ag} / \mathrm{AgCl}$ electrode, and a temperature sensor (PT1000). As seen in Fig. 1, the temperature sensor, $\mathrm{pH}$ glass electrode, and $\mathrm{Ag} / \mathrm{AgCl}$ electrode were placed in the storage tank. The tungsten oxide electrode was immersed and fixed in a flow cell with an inner diameter of $10 \mathrm{~mm}$. The solution in the storage tank was pumped to the flow cell by a peristaltic pump (BT50S, Baoding LeadFluid Technology, China), and then flowed back to the storage tank. The adjustable range of average flow velocity in the flow cell was 0.08 to $40 \mathrm{~mm} / \mathrm{s}$ with a resolution of $0.08 \mathrm{~mm} / \mathrm{s}$. The PT1000 senor was the temperature sensor used to continuously monitor the temperature of solution. The tungsten oxide electrode and $\mathrm{pH}$ glass electrode were 
used as an indicator electrode separately, and the $\mathrm{Ag} / \mathrm{AgCl}$ electrode was used as a reference electrode, the open circuit potentials between the indicator and reference electrodes were measured. The potentiometric measurements were performed on an electrochemical station (CHI760, $\mathrm{CH}$ Instruments, USA). All the data presented below have been compensated according to the temperature characteristics of the tungsten oxide electrode.

\section{Results and discussion}

\subsection{Mechanism and characteristics of flow sensor based on the tungsten oxide} electrode

The tungsten oxide electrode has a bilayer structure consisting of a compact inner layer of $\mathrm{WO}_{3}$ and a loosely bound outer hydrated layer of $\mathrm{WO}_{3} \cdot \mathrm{xH}_{2} \mathrm{O}$. Lillard et al. [16] has reported a corresponding decrease in the thickness of the surface layer under the hydrodynamic effect, our previous work also demonstrated a dramatic and positive change in the open circuit potential of the tungsten oxide electrode under the hydrodynamic effect [17].

In order to investigate the mechanism of flow sensor, the change of potentials were measured for the flow velocity of $1.6 \mathrm{~mm} / \mathrm{s}$ in solutions of different $\mathrm{pH}$ which contain the point of zero charge (pzc). The experimental result was seen in the Fig. 2. When the tungsten oxide electrode was immersed in the solution of $\mathrm{pH}$ above the point of zero charge, tungsten oxide sensor surface was charged negatively. A flow induced streaming potential at the solution/electrode interface comprising deprotonated terminal hydroxyl groups from the hydrated tungsten oxide. Therefore, 
the potential of tungsten oxide electrode would shift positively in the flowing fluid. However, the potential of tungsten oxide electrode would shift negatively in the solution of $\mathrm{pH}$ below pzc as the terminal hydroxyl groups of the hydrated oxide surface become protonated. The experiment strongly suggests that potentiometric response behavior induced by flow was based on streaming potential.

To explore characteristics of flow sensor based on the tungsten oxide electrode, the potentials of the tungsten oxide and $\mathrm{pH}$ glass electrodes in the buffer solution of pH 6.86 were simultaneously monitored in realtime. Once the open circuit potentials stabilized, the flow velocity was changed using the digitally tunable peristaltic pump, and then maintained for $\sim 180 \mathrm{~s}$ in the same state. These flow velocities were $0.4,0.8$, 1.6, 3.2, 6.4, 12.8, and $25.6 \mathrm{~mm} / \mathrm{s}$. As seen in Fig. 3, the open circuit potential of the tungsten oxide electrode shifts positively with increasing flow velocity, and remains stable after a short rising time. The inset of Fig. 3 shows the rising time and fluctuation noise, which are $\sim 40 \mathrm{~s}$ and $\sim 0.15 \mathrm{mV}$ respectively. Meanwhile, the open circuit potential of the $\mathrm{pH}$ glass electrode barely changed and reflected the accurate $\mathrm{pH}$ value.

The tungsten oxide electrode could be used to measure ultralow flow velocities because its open circuit potential shifted positively by $\sim 10 \mathrm{mV}$ even when the flow velocity was $0.4 \mathrm{~mm} / \mathrm{s}$. Moreover, the gaps in potentials were clearly observable for each of the flow velocities tested, and the stability of open circuit potentials was satisfactory for any given flow velocity. Notably, the large fluctuations in open circuit potentials for low flow velocities were caused by the inherent property of the 
peristaltic pump, and the fluctuations disappeared for relatively high flow velocities.

\subsection{Calibration of flow sensor}

In order to explore the relationship between open circuit potential and flow velocity, both the slope of E vs. the flow velocity curve and the scatters of the measured potential values are presented in Fig. 4(A). The potential values presented for flow velocities of 0.4 and $0.8 \mathrm{~mm} / \mathrm{s}$ were the average points in the peaks of their respective waves shown in Fig. 3, and the open circuit potentials are seen to be linear relative to the logarithmic flow velocities $\left(R^{2}=0.99\right)$. The error bars were acquired from four repetitions of the experiment completed on four consecutive days. The results of these repetitions are close, indicating that this flow sensor exhibits excellent reproducibility for the measurement of ultralow flow velocity. Moreover, the ratio between measurable maximum and minimum flow velocity surpasses $60: 1$, which is a large dynamic range for the measurement of ultralow liquid flow velocity.

Tungsten oxide electrode exhibits a near-Nernstian behavior with respect to $\mathrm{pH}$; therefore, we carried out the same experiments in buffer solutions of $\mathrm{pH} 4$ and $\mathrm{pH}$ 9.18. These experiments produced similar linear curves, as can be seen in Fig. 4(B) and Fig. 4(C). When the flow velocity surpasses $25.6 \mathrm{~mm} / \mathrm{s}$, the positive shift in open circuit potential starts to get smaller, indicating that the decrease in the thickness of the surface layer would reach a critical limit. Thus, the tungsten oxide flow sensor is not suitable for the measurement of high flow velocity, because it is difficult for open circuit potential to change in such velocities.

The system described herein yields information on liquid flow velocity, $\mathrm{pH}$, and 
temperature, and the open circuit potentials shown have been compensated according to the temperature characteristics of tungsten oxide electrode. The relationships between open circuit potential and $\mathrm{pH}$ in four kinds of flow velocity are shown in Fig. 5. The ultralow liquid flow velocity could be measured by using the open circuit potential of tungsten oxide electrode together with the solution's $\mathrm{pH}$ value. Notably, the open circuit potentials change more easily in the alkaline solution than in the acidic solution. In other words, open circuit potential has a higher sensitivity in alkaline solutions than in acidic solutions. Therefore, alkaline solutions are more suitable for the measurement of ultralow flow velocity.

\subsection{Merits of flow sensor based on tungsten oxide electrode}

Using a tungsten oxide electrode for measurement of flow velocities has many advantages. First, this kind of flow sensor can measure ultralow liquid flow velocities and can be used in open channels or various kinds of pipelines. The sensor does not have strict requirements for the location of installation in liquids, and miniaturization and portability can be achieved easily.

Second, the tungsten oxide flow sensor avoids the signal stimulus that often plagues electromagnetic, ultrasonic, and thermal flow sensors. Thus, it is possible to develop a product with low power consumption, and a battery can be used as the energy source instead of switching power supply. Furthermore, the value of flow velocity is readily obtainable using the open circuit potential, $\mathrm{pH}$ value, and temperature, without the need for complex signal processing and calculation.

Third, pure tungsten is a cheap metal, and the tungsten oxide used for the sensor 
is easy to prepare. Techniques for the measurements of open circuit potential, $\mathrm{pH}$ value, and temperature have matured sufficiently because of the importance of these parameters in various kinds of process controls. Thus, low cost can also be achieved using tungsten oxide electrode for the measurement of ultralow liquid flow velocity.

\section{Conclusion}

In this study, we successfully utilized a tungsten oxide electrode for the measurement of ultralow liquid flow velocity based on streaming potential, using a $\mathrm{pH}$ glass electrode and temperature sensor to compensate for changes in open circuit potential induced by $\mathrm{pH}$ and temperature, respectively. The results demonstrated that the open circuit potential of the tungsten oxide electrode exhibits good linearity and repeatability with respect to logarithmic flow velocity. The ratio between the measurable maximum and minimum flow velocity surpassed 60:1, which is a large dynamic range for the measurement of ultralow liquid flow velocity. In conclusion, the flow sensor provides a cheap, simple, adaptable, and energy-saving solution for the measurement of ultralow liquid flow velocity.

\section{Acknowledgments}

The study was supported by National High Technology Research and Development Program of China (863 Program) (Project No.2014AA06A504) and the Major Scientific and Technological Projects of Zhejiang Bureau of Quality and Technical Supervision (Project No. 20110105). We also thank Yujie Yang at China Jiliang University for many fruitful discussions.

\section{Reference}


[1] A. Michalski, J. Starzynski, S. Wincenciak, Optimal design of the coils of an electromagnetic flow meter, IEEE Transactions on Magnetics, 34 (1998) 2563-2566.

[2] L.C. Lynnworth, Y. Liu, Ultrasonic flowmeters: Half-century progress report, 1955-2005, Ultrasonics, 44 (2006) E1371-E1378.

[3] M.A. Atmanand, M.S. Konnur, A novel method of using a control valve for measurement and control of flow, Ieee Transactions on Instrumentation and Measurement, 48 (1999) 1224-1226.

[4] H.J. Zhang, Y.M. Huang, Z.Q. Sun, A study of mass flow rate measurement based on the vortex shedding principle, Flow measurement and instrumentation, 17 (2006) 29-38.

[5] A. Venugopal, A. Agrawal, S.V. Prabhu, Influence of blockage and shape of a bluff body on the performance of vortex flowmeter with wall pressure measurement, Measurement, 44 (2011) 954-964. [6] R.C. Baker, Turbine flowmeters: II. Theoretical and experimental published information, Flow measurement and instrumentation, 4 (1993) 123-144.

[7] R.J. Rodrigues, R. Furlan, Design of microsensor for gases and liquids flow measurements, Microelectronics Journal, 34 (2003) 709-711.

[8] M. Takamoto, H. Ishikawa, K. Shimizu, H. Monji, G. Matsui, New measurement method for very low liquid flow rates using ultrasound, Flow measurement and instrumentation, 12 (2001) 267-273. [9] T.X. Dinh, V.T. Dau, S. Sugiyama, P.H. Pham, Fluidic device with pumping and sensing functions for precise flow control, Sensors and Actuators B: Chemical, 150 (2010) 819-824.

[10] Y. Kikutani, K. Mawatari, K. Katayama, M. Tokeshi, T. Fukuzawa, M. Kitaoka, et al., Flowing thermal lens micro-flow velocimeter, Sensors and Actuators B: Chemical, 133 (2008) 91-96.

[11] J.G. Santiago, S.T. Wereley, C.D. Meinhart, D.J. Beebe, R.J. Adrian, A particle image velocimetry system for microfluidics, Experiments in Fluids, 25 (1998) 316-319. 
[12] V. Lien, F. Vollmer, Microfluidic flow rate detection based on integrated optical fiber cantilever, Lab Chip, 7 (2007) 1352-1356.

[13] Q. Zhang, W.Z. Ruan, H. Wang, Y.Z. Zhou, Z.Y. Wang, L.T. Liu, A self-bended piezoresistive microcantilever flow sensor for low flow rate measurement, Sensors and Actuators A: Physical, 158 (2010) 273-279.

[14] M. Durán-Sánchez, G. Beltrán-Pérez, J. Castillo-Mixcóatl, S. Muñoz-Aguirre, M. Méndez-Otero, Experimental study of the fiber laser output intensity behavior and its application to a water flow sensor, Sensors and Actuators B: Chemical, 123 (2007) 816-821.

[15] H. Park, S.-H. Choa, Measurement of liquid flow rate by self-generated electrokinetic potential on the microchannel surface of a solid, Sensors and Actuators A: Physical, 208 (2014) 88-94.

[16] R. Lillard, G. Kanner, D. Butt, The nature of oxide films on tungsten in acidic and alkaline solutions, Journal of The Electrochemical Society, 145 (1998) 2718-2725.

[17] Y.Z. Wen, X.P. Wang, Characterization and application of a metallic tungsten electrode for potentiometric pH measurements, Journal of Electroanalytical Chemistry, 714 (2014) 45-50.

[18] I.A. Pašti, T. Lazarević-Pašti, S.V. Mentus, Switching between voltammetry and potentiometry in order to determine $\mathrm{H}^{+}$or $\mathrm{OH}^{-}$ion concentration over the entire $\mathrm{pH}$ scale by means of tungsten disk electrode, Journal of Electroanalytical Chemistry, 665 (2012) 83-89.

\section{Author Biographies:}

Yizhang Wen is a PhD student in Zhejiang University, Hangzhou, China (2012 now). Research interests are electrochemical sensors, optical sensors and the application in monitoring water environment. 
Xiaoping Wang received her $\mathrm{PhD}$ in 2004 from Zhejiang University, Hangzhou, China, holding the post of deputy director of the department of optical engineering, Zhejiang University. Her interests cover SPR sensor, electrical tongue, Raman spectroscope measurements, and the application in monitoring water environment and food quality.

Peijun Cai received her Master in 2013 from Zhejiang University, Hangzhou, China. Her interests cover electrical tongue, electrical design, and the application in monitoring water environment and food quality.

Bing Zhang is a PhD student in Zhejiang University, Hangzhou, China (2011 now). Her interests cover E-SPR sensor, Raman spectroscope measurements, and the application in monitoring water environment and food quality.

\section{Figure captions}

Fig. 1 Experimental setup.

Fig. 2 The change of potentials for the flow velocity of $1.6 \mathrm{~mm} / \mathrm{s}$ in solutions of different $\mathrm{pH}$.

Fig. 3 Simultaneous real-time monitoring of potentials of tungsten oxide electrode and $\mathrm{pH}$ glass electrode in a buffer solution of $\mathrm{pH} 6.86$.

Fig. 4 Open circuit potentials with respect to flow velocities in a buffer solution of (A) $\mathrm{pH} 6.86$, (B) $\mathrm{pH} 4$, and (C) $\mathrm{pH} 9.18$.

Fig. 5 Calibration of flow sensor based on tungsten oxide electrode. 


\section{Figures}

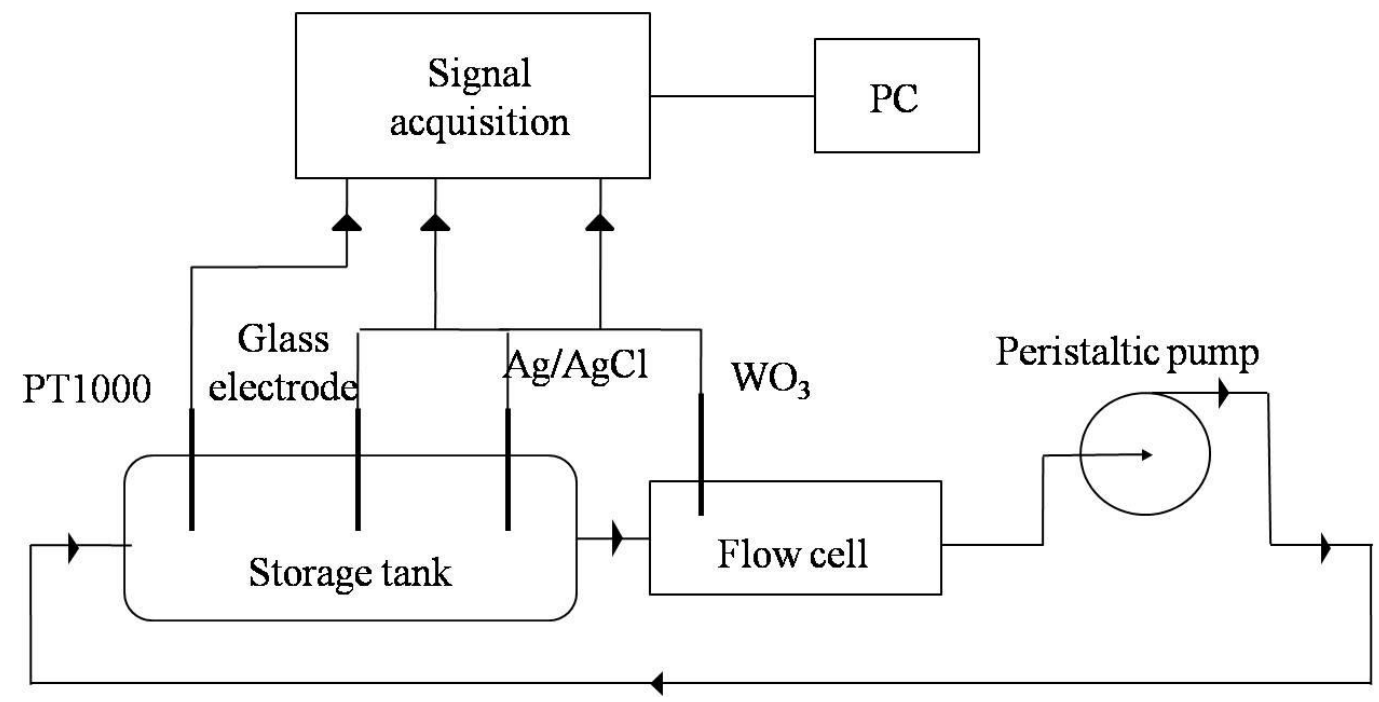

Fig. 1

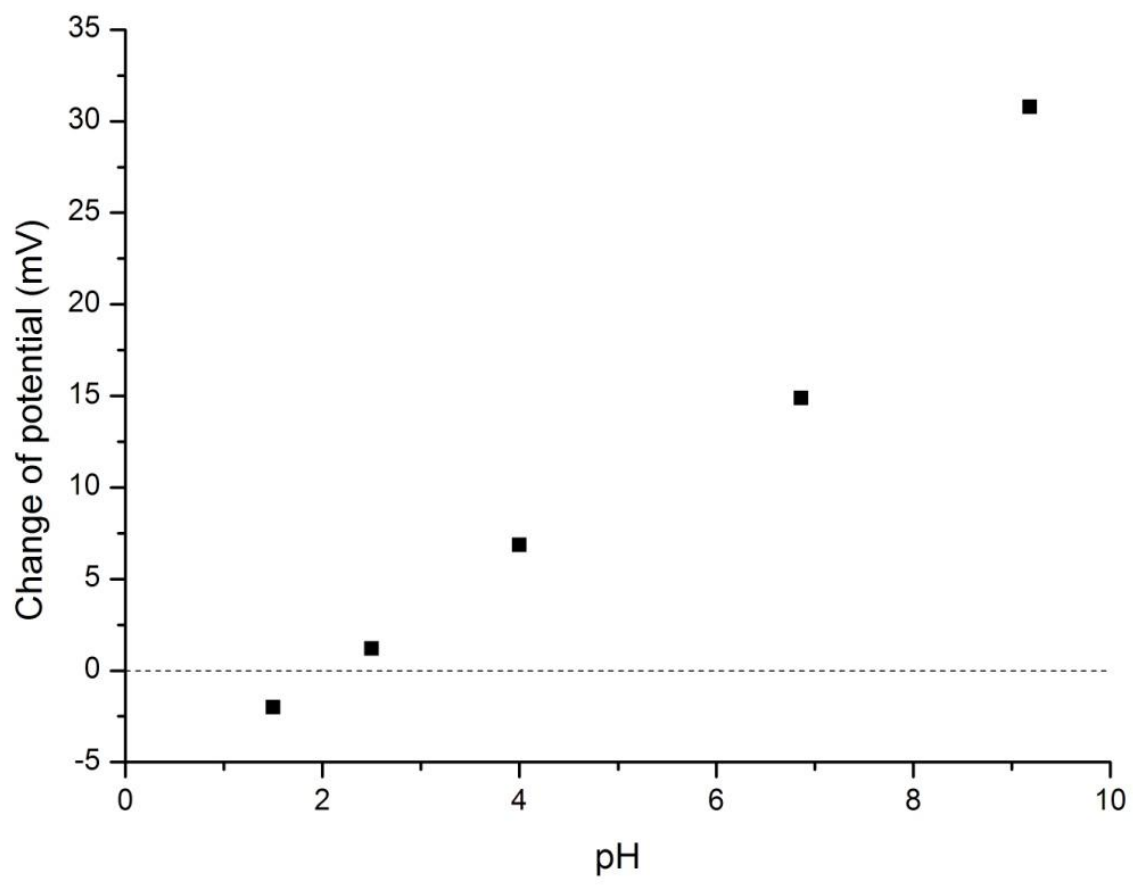

Fig. 2 


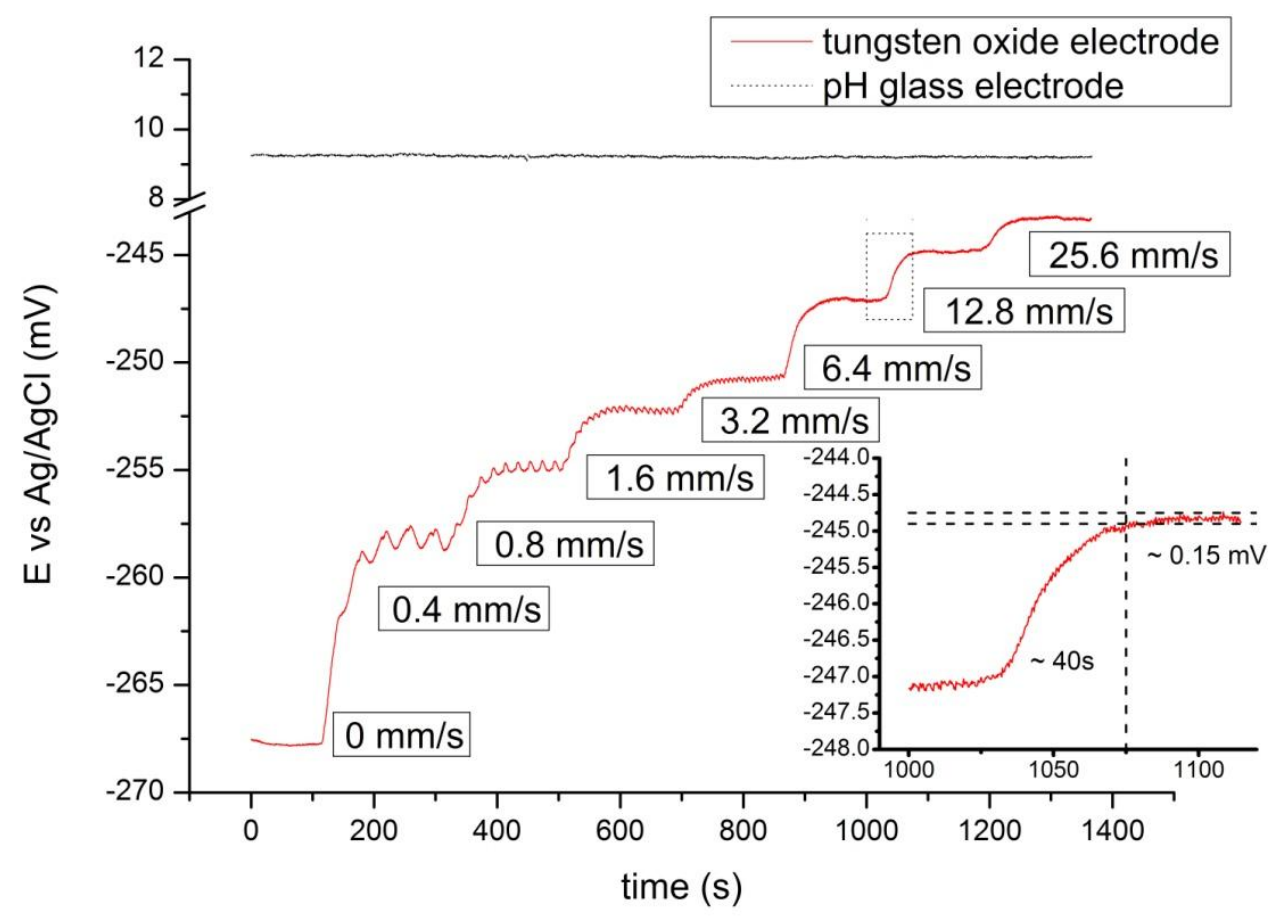

Fig. 3

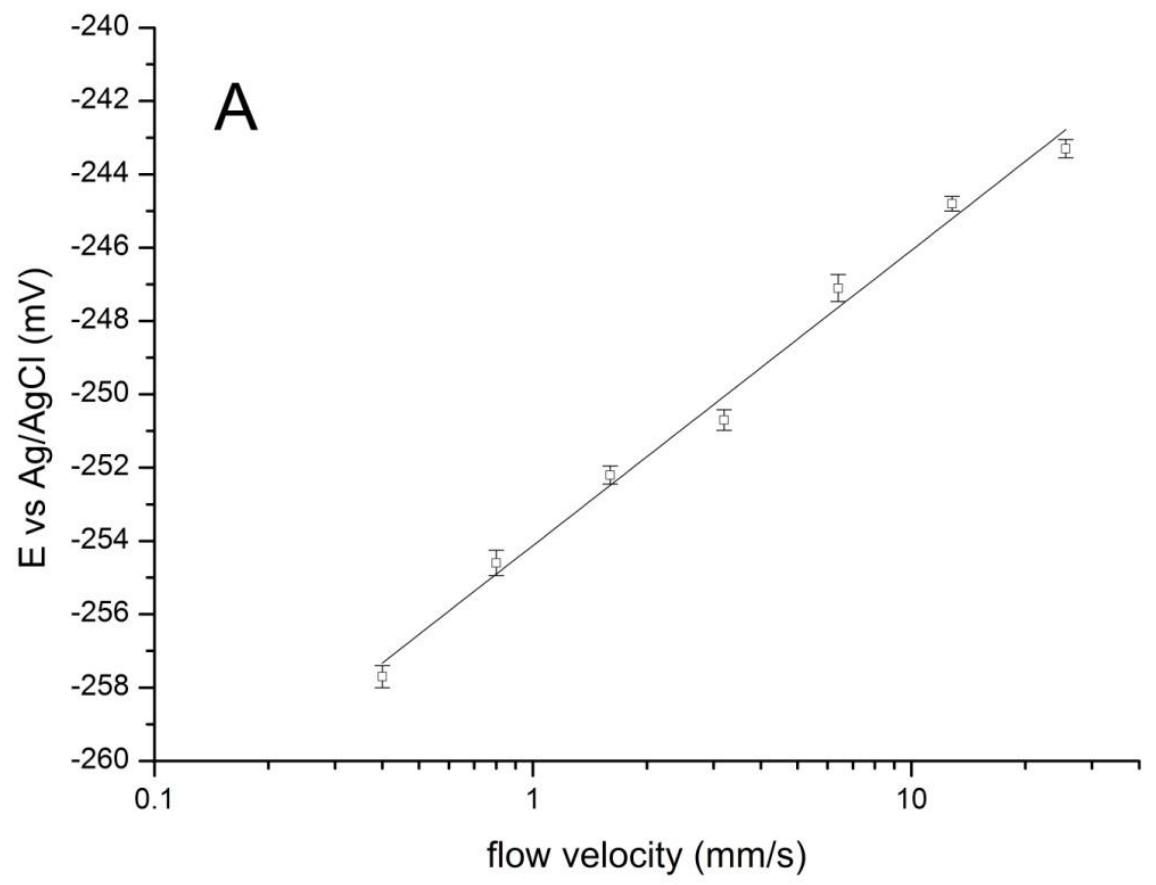



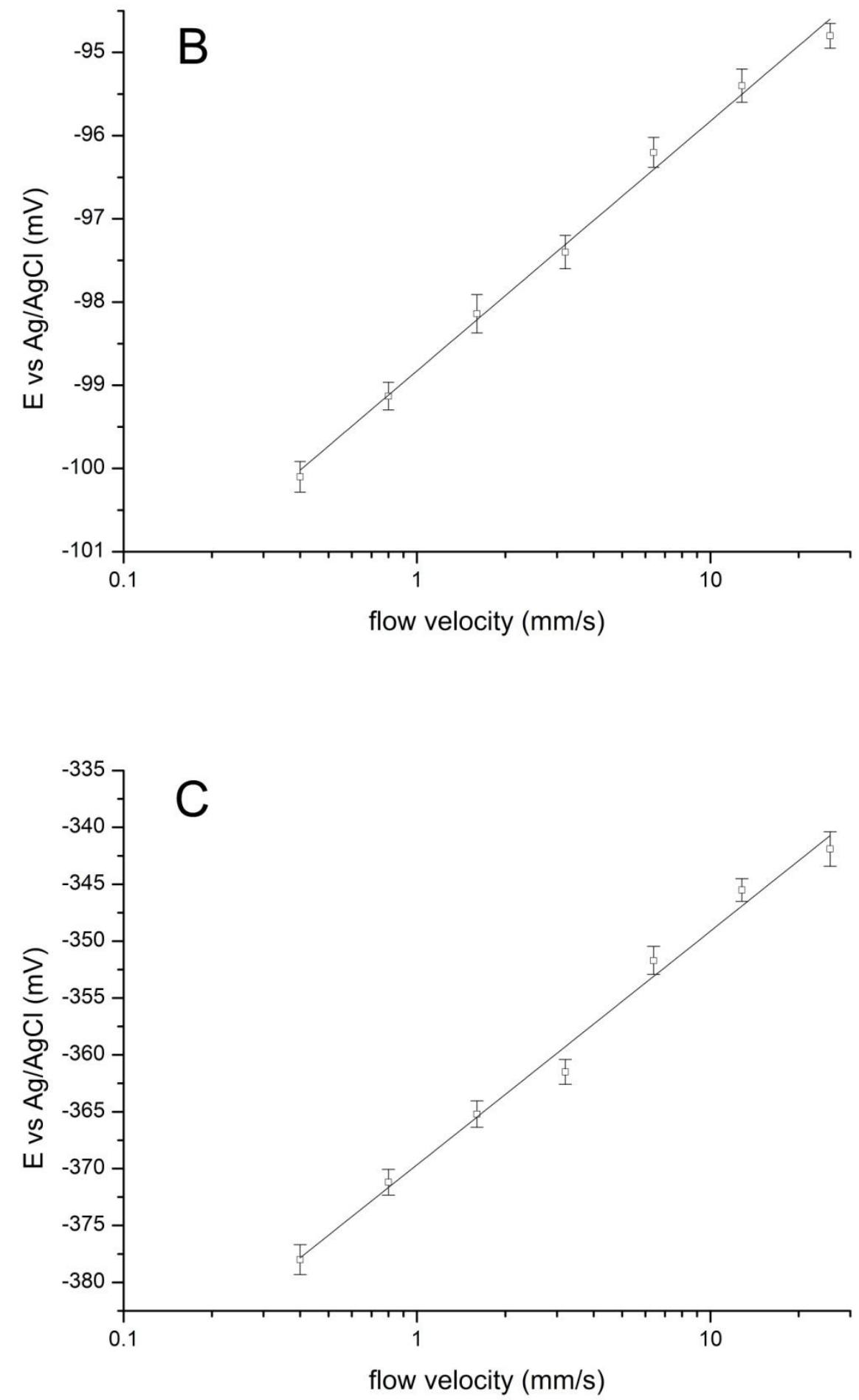

Fig. 4 


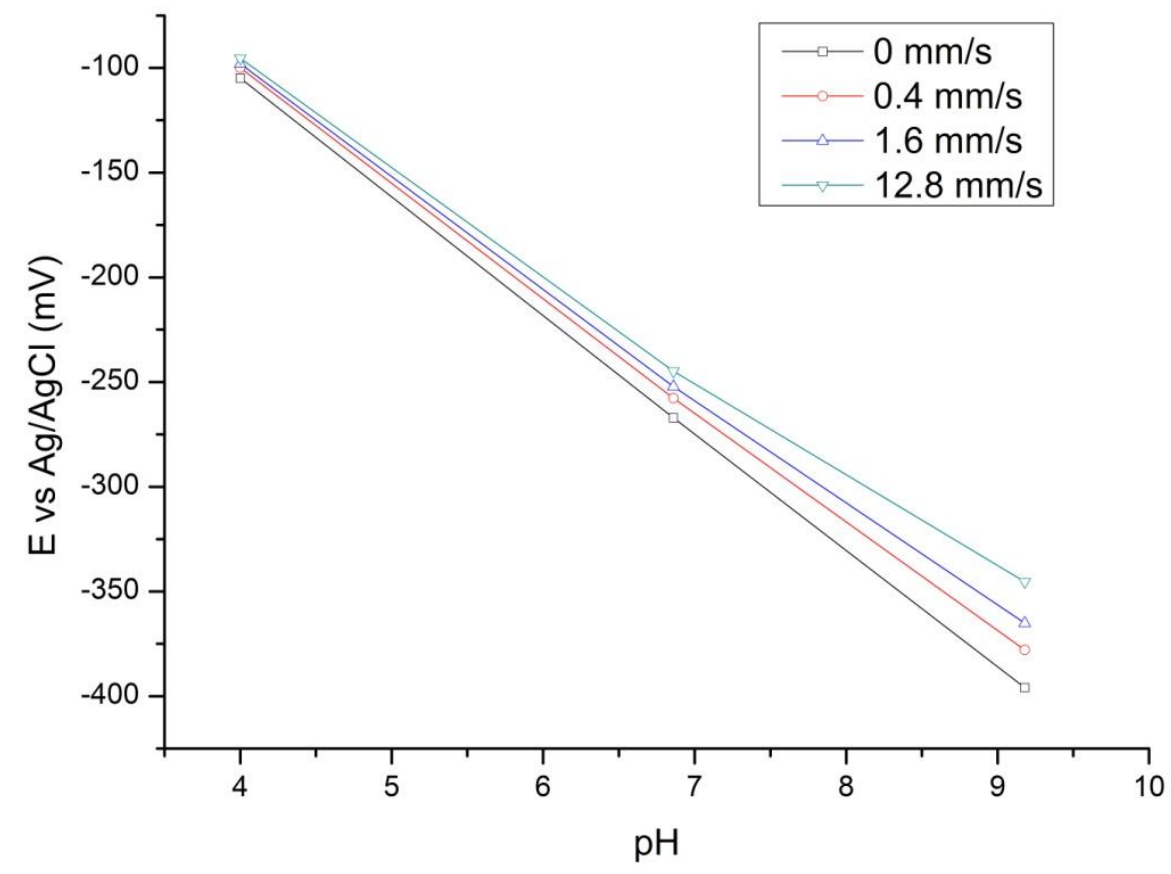

Fig. 5 


\section{Author Biographies:}

Yizhang Wen is a PhD student in Zhejiang University, Hangzhou, China (2012 now). Research interests are electrochemical sensors, optical sensors and the application in monitoring water environment.

Xiaoping Wang received her PhD in 2004 from Zhejiang University, Hangzhou, China, holding the post of deputy director of the department of optical engineering, Zhejiang University. Her interests cover SPR sensor, electrical tongue, Raman spectroscope measurements, and the application in monitoring water environment and food quality.

Peijun Cai received her Master in 2013 from Zhejiang University, Hangzhou, China. Her interests cover electrical tongue, electrical design, and the application in monitoring water environment and food quality.

Bing Zhang is a $\mathrm{PhD}$ student in Zhejiang University, Hangzhou, China (2011 now). Her interests cover E-SPR sensor, Raman spectroscope measurements, and the application in monitoring water environment and food quality. 